\title{
Strategies To Improve Linkage To HIV Care In Urban Areas Of Sub-Saharan Africa: A Systematic Review
}

This article was published in the following Dove Press journal:

HIVIAIDS - Research and Palliative Care

\author{
Kwadwo Koduah Owusu (D) \\ Raphael Adu-Gyamfi (D) ${ }^{2}$ \\ Zamzam Ahmed' \\ 'School of Life and Medical Sciences, \\ University of Hertfordshire, \\ Hertfordshire, UK; ${ }^{2}$ National AIDS/STI \\ Control Programme, Ghana Health \\ Service, Korle-Bu, Accra, Ghana
}

\begin{abstract}
Of the 37 million people estimated to be living with HIV globally in 2017, about 24.7 million were in the sub-Saharan Africa region, which has been and remains worst affected by the epidemic. Enrolment of newly diagnosed individuals into care in the region, however, remains poor with up to $54 \%$ not being linked to care. Linkage to care is a very important step in the HIV cascade as it is the precursor to initiating antiretroviral therapy (ART), retention in care, and viral suppression. A systematic review was conducted to gather information regarding the strategies that have been documented to increase linkage to care of Persons living with HIV(PLHIV) in urban areas of sub-Saharan Africa. An electronic search was conducted on Scopus, Cochrane central, CINAHL Plus, PubMed and OpenGrey for linkage strategies implemented from 2006. A total of 189 potentially relevant citations were identified, of which 7 were eligible for inclusion. The identified strategies were categorized using themes from literature. The most common strategies included: health system interventions (i.e. comprehensive care, task shifting); patient convenience and accessibility (i.e. immediate CD4 count testing, immediate ART initiation, community HIV testing); behavior interventions and peer support (i.e. assisted partner services, care facilitation, mobile phone appointment reminders, health education) and incentives (i.e. non-cash financial incentives and transport reimbursement). Several strategies showed favorable outcomes: comprehensive care, immediate CD4 count testing, immediate ART initiation, and assisted partner services. Assisted partner services, same day home-based ART initiation, combination intervention strategies and point-of-care CD4 testing significantly improved linkage to care in urban settings of sub-Saharan African region. They can be delivered either in a health facility or in the community but should be facilitated by health workers. There is, however, the need to conduct more linkage-specific studies in the sub-region.
\end{abstract}

Keywords: linkage to care, human immunodeficiency virus, acquired immune deficiency syndrome, urban, sub-Saharan Africa, adults

\section{Introduction}

\section{Epidemiology Of HIV}

The HIV epidemic is one of the major public health threats with over 70 million persons being infected with the virus since it was identified, and has resulted in about 35 million deaths. Globally, about 37 million people were estimated to be living with HIV in 2017. ${ }^{1}$ In 2014, about 24.7 million persons were living with HIV in sub-Saharan Africa, which has been worst affected by the epidemic. ${ }^{2}$

Globally, an estimated $0.8 \%$ of adults aged 15 to 49 years are living with HIV. ${ }^{3}$ However, the prevalence varies considerably between countries and regions, with the sub-Saharan African region being the worst affected with about $4 \%$ of adults
Correspondence: Raphael Adu-Gyamfi National AIDS/STI Control Programme, Ghana Health Service, Korle-bu, Accra, Ghana

Tel +233267075458

Email ralphgyamfi@gmail.com 
living with $\mathrm{HIV}^{3}$ However, there has been a significant expansion of program coverage in the region with more than 19 million people accessing antiretroviral therapy (ART) over the past ten years. ${ }^{4}$ This has resulted in a substantial reduction in HIV-related morbidity and mortality along with a reduction in the rate of new infections. Since 1995, ART has prevented an estimated 4.8 million deaths in sub-Saharan Africa alone. ${ }^{2}$

The prevalence of HIV in the sub-Saharan Africa region also differs among various subgroups with the urban population generally having higher prevalence as compared to rural dwellers. ${ }^{5}$ Moreover, there are significant differences between the rural and urban settings which may require different approaches.

\section{Efforts Towards Epidemic Control}

Despite the significant gains made in the HIV response, the epidemic is yet to be controlled as it is impossible to end the HIV pandemic without providing treatment to all PLHIV. $^{2}$ In order to help end the HIV epidemic, all countries are expected to meet the UNAIDS 90-90-90 targets which require that by the year 2020;

- $90 \%$ of all persons living with HIV will know their HIV status,

- $90 \%$ of all persons with diagnosed HIV infection will receive sustained ART and

- $90 \%$ of all persons receiving ART will have viral suppression. $^{6}$

For countries to achieve the second and third 90s, there is the need to ensure that all persons diagnosed with the infection are successfully linked to ART care.

\section{Linkage To Care}

Prompt linkage of persons living with HIV to care is critical to ending AIDS as a public health threat. ${ }^{7}$ Linkage to care is defined as a patient who receives clinical HIV care following diagnosis through at least one of the following:

- a clinical assessment or WHO staging,

- immunologic assessment (CD4 count),

- virologic test (Viral Load) or

- receiving $\mathrm{ART}^{8}$

This is a very important step in the HIV cascade as it is the precursor to initiating ART and retention in care for viral suppression. The evidence available indicates that delays in the linkage to care results in delayed receipt of ART, quicker disease progression, and increased mortality. ${ }^{7,9,10}$ Also, ART significantly reduces HIV transmission rates and is important in preventing new infections. ${ }^{11-13}$ Therefore, successful linkage to care is undoubtedly critical in fighting the epidemic.

Various HIV programmes around the world have recorded varying rates of linkage from less than $50 \%$ after six months of HIV diagnosis to $90 \%$ being ever linked. ${ }^{14-19}$ Results from Western countries have been relatively very high with previous studies reporting $85 \%$ for the World Health Organization (WHO) European region, $84 \%$ in the United States of America, 73\% for Canada and 90\% in Australia. ${ }^{7,9,10}$ However, very poor results have been obtained in sub-Saharan Africa as the majority of infected persons start ART very late and in the advanced stages of infection. ${ }^{18,20}$ A synthesis of available literature from subSaharan Africa indicated that $54 \%$ of HIV positive persons who were not linked. ${ }^{18,21,22}$ This results in high mortality soon after starting therapy. ${ }^{23,24}$

\section{Strategies To Improve Linkage To Care}

Several strategies have been suggested to improve linkage to care for newly diagnosed persons living with HIV around the world. A systematic review by Govindasamy et al. ${ }^{25}$ in low and middle-income countries across the globe grouped the strategies under the following domains with examples beneath:

1. Health system interventions

- Task shifting- This includes sharing and shifting of HIV service provisions tasks among professional health workers and lay providers. ${ }^{25}$

- Integration of HIV services into routine care. ${ }^{25}$

- Comprehensive HIV care including improved referrals, teaching, and communication ${ }^{25}$

2. Patient convenience and accessibility

- Immediately referring PLHIV to care and providing CD4 count test and same day initiation of ART. ${ }^{11,26-28}$ Where this is not possible, the waiting times for the initial appointment should be reduced. $^{11}$

- Home-based initiation of ART after HIV diagnosis. $^{25}$

3. Behavior interventions and peer support

- The use of trained patient navigators and case managers to support persons who test HIV positive to be linked to care. ${ }^{11,26,29}$ 
- Active search for patients who miss clinic appointments. This includes community outreach for patients. ${ }^{11,26}$

- Assisted partner services. This includes various services that may be offered to persons living with HIV and their newly diagnosed partners to assist in linking them to care..$^{11,26,30,31}$

4. Use of incentives

- Providing financial assistance and incentives to support PLHIV to attend clinic appointments. ${ }^{11,26,32}$

Some reviews have been conducted in sub-Saharan Africa to improve service delivery across the cascade of care. However, there is a paucity of linkage-specific reviews focussing on the urban settings of sub-Saharan Africa. Moreover, many studies have focussed on the factors that reduce linkage, with a limited focus on the strategies that can be used to address the challenges. There is, therefore, the need to synthesize evidence from primary studies that looked at improving linkage rates in the urban settings of the region to help develop recommendations for program managers and service providers.

The aim of this systematic review is to summarize available data on the strategies that have been documented to increase linkage to care of PLHIV in urban areas of subSaharan Africa.

\section{Research Methodology/Methods And Materials}

A systematic review was conducted between October 2018 and December 2018 as follows:

\section{Inclusion Criteria}

Studies that fulfilled all the conditions below were eligible for inclusion:

(a) Population- Adults defined as 15 years or older by the UNAIDS, ${ }^{33}$ diagnosed with HIV and living in urban Sub-Saharan Africa.

(b) Intervention- The review investigated the strategies that improved linkage to care so the search was not limited to any particular intervention.

(c) Comparison- Studies that compared various strategies with either no care or standard care as defined by the various studies were eligible for inclusion (d) Outcomes- The primary outcome measure for the review was the impact of the interventions on linkage to care.

Also, the following study designs were considered for inclusion:

- Randomized controlled trials

- Retrospective cohort studies

- Prospective cohort studies

\section{Exclusion Criteria}

Included studies that were not published in the English language, conducted outside the 48 countries in subSaharan Africa, ${ }^{34}$ not conducted in urban settings and not among adult HIV positive patients as defined previously were excluded from the analysis. Studies not comparing strategies to usual or routine or no care and not measuring linkage as an outcome were not included. Study protocols and pilot studies, as well as studies with no full text available, were also excluded from the analysis. In addition, studies published before 1st January 2006 were also not used. This is because they were conducted before the WHO patient monitoring guidelines for management of HIV was developed. ${ }^{35}$ This policy is essential as it indicated the basic requirements for standard HIV care and treatment.

\section{Search Strategy}

The search terms were derived after the review question as stated in the research proposal, was separated into the Population, Intervention, Comparison, Outcome and Study design (PICOS). Medical subject headings $(\mathrm{MeSH})$ were identified for the key terms indicated in Table 1 and combined with free text using Boolean operators. The search strategy was piloted in PubMed and adapted to the other four databases, CINAHL plus, Scopus, Cochrane Central Register of Controlled Trials and OpenGrey based on their complexity and vocabulary. The database search strategy can be found in Appendix A. The reference lists of all included articles were also screened further for relevant publications. Articles with relevant titles were reviewed for possible inclusion.

\section{Reference Management And Study Selection}

The results from the database search and the review of the reference lists were exported to RefWorks and the duplicates were removed. The titles and abstracts of the 
Table I The Key Search Terms

\begin{tabular}{|c|c|}
\hline Stage & Key Terms \\
\hline Population & $\begin{array}{l}\text { - Adults } \\
\text { - HIV positive } \\
\text { - Urban } \\
\text { - Sub-Saharan Africa }\end{array}$ \\
\hline Intervention & Strategies to improve linkage to care \\
\hline Outcome & Improved linkage rates \\
\hline Types Of Study & $\begin{array}{l}\text { - Randomized controlled trials } \\
\text { - Retrospective cohort studies } \\
\text { - Prospective cohort studies }\end{array}$ \\
\hline
\end{tabular}

remaining references were screened for relevant studies using the inclusion criteria. The full articles of abstracts that did not have enough information were reviewed. Abstracts of the potentially relevant studies were assessed by a second reviewer and full texts of the final list of studies retrieved and examined for compliance with eligibility criteria. Disagreements in the abstract selection was resolved by a third reviewer.

\section{Risk Of Bias Assessment}

To guide the interpretation of intervention study findings, there was a need to ensure their internal validity was not compromised, and the likelihood of reporting false intervention effects reduced. The risk of bias in the included randomized controlled trials was assessed using the Cochrane's Collaboration tool for assessing the risk of bias. The Newcastle-Ottawa scale for observational studies was used to assess the risk of bias in the included cohort studies. ${ }^{36}$ The assessment was done looking at sequence generation, concealment of allocation, the blinding of participants and personnel, incomplete outcome data and selective outcome reporting as indicated in the tool.

\section{Data Extraction Plan}

In order to standardize the data extraction process, limit bias and ensure that all the relevant details were identified, a data extraction form was used. The recommendations in the book "The checklist to improve reporting of groupbased behavior change interventions" was used as a guide to design the form ${ }^{37}$ This checklist was used because apart from including suggestions on intervention content, intervention design and the characteristics of participants, it has high inter-rater reliability for identifying reporting elements (with 95\% agreement, Mean $\mathrm{AC} 1=0.89$ ). ${ }^{37}$
A second person reviewed the data extracted from the selected studies.

The data extracted included:

- Lead author

- Year of publication

- Type of study

- Participants

- Strategies

- Control

- Number of participants

- Number of interventions

- Site (health facility or community)

- Follow up period (months)

- Facilitator characteristics (health worker or lay provider)

- Primary measure

- Primary outcome

- Other outcomes

\section{Data Analysis}

The extracted data was categorized using themes from the systematic review by Govindasamy et al. ${ }^{25}$ to compare the effect of the strategy on the primary outcome and other outcomes in the comparison arm.

\section{Results/Findings}

A total of 189 references were retrieved from the database search and the review of the references in the initially selected studies. This initial list included 63 duplicates. After assessment of the titles and abstracts, 19 were obtained for full-text retrieval. Applying the exclusion criteria to the full texts resulted in the exclusion of 12 more publications. The reasons for exclusion are indicated in Appendix B. The risk of bias in the remaining 7 studies was assessed and can be found in Appendix C. The study selection process is as depicted in Figure 1 below.

\section{Participants/Setting}

A total of 24,810 participants, aged 15 years and older, were represented in the 7 studies included in this review. The majority were females comprising $59 \%^{38}$ to $100 \%{ }^{39}$ of the study population. The included studies were conducted in Kenya, ${ }^{39}$ South Africa ${ }^{40}$ Tanzania $^{41}$ Swaziland $^{38}$ Mozambique, ${ }^{42}$ Zambia $^{43}$ and Lesotho. ${ }^{44}$ One was conducted among antenatal clients ${ }^{39}$ and the rest among general patients. Between $268^{42}$ and $15,557^{45}$ participants were involved in each study. 


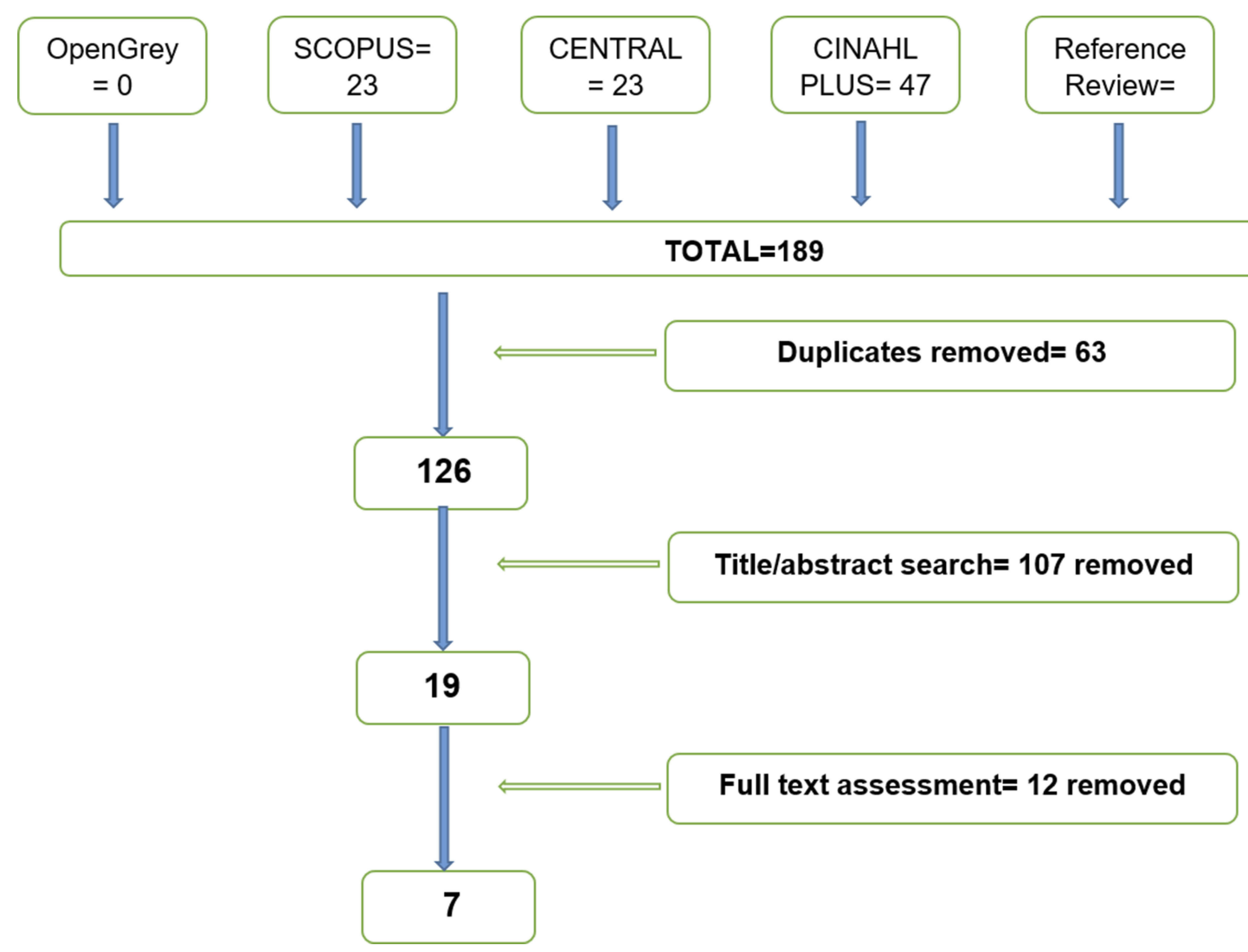

Figure I Flowchart of the study selection process.

The comparison groups that were used included waiting list control, ${ }^{39}$ standard care, ${ }^{38,40,42,44}$ facility testing ${ }^{41}$ and non-HIV self-testing. ${ }^{45}$ Two of the strategies ${ }^{38,42}$ were delivered in health facilities and the rest delivered in community settings. All the strategies were delivered by healthcare workers except one. ${ }^{45}$ Two of the strategies were delivered as combination interventions ${ }^{38,42}$ and the rest as individual interventions.

\section{Summary Of Individual Studies}

The studies included in the review are made up of a prospective mixed-method cohort study, ${ }^{41}$ cluster randomized trials, ${ }^{38,39,42,45}$ and randomized trials. ${ }^{40,44}$ The details of the studies used are indicated in Table 2.

\section{Impact Of Strategies On Linkage}

The strategies that were used in the studies are listed under the main domains:

\section{Health System Strategies}

- Comprehensive HIV care
Two of the studies ${ }^{38,42}$ provided comprehensive post-diagnosis care to newly diagnosed patients. Elul et al aimed to simplify the clinic flow and encourage linkage to and retention in care through the provision of point-of-care (POC) CD4 testing at the time of diagnosis, accelerated ART initiation, short message service (SMS), health messages and appointment reminders in Mozambique. ${ }^{42}$ McNairy et al also aimed to improve linkage in Swaziland through the provision of POC CD4 testing at the time of an HIV-positive test, accelerated ART initiation for treatment-eligible participants, mobile phone appointment reminders and health education packages. ${ }^{38}$ Whilst there was a significant improvement in linkage in the intervention group over the controls in the Mozambique study ( $\mathrm{RR}=1.48, \mathrm{CI}: 0.93 \pm 2.35, p=0.09),{ }^{42}$ there was no significant difference in the linkage between the comparison groups in Swaziland $(\mathrm{RR}=1.48, \mathrm{CI}: 1.18 \pm 1.86$, $p=0.002) .{ }^{38}$ In both studies, however, there was a significant improvement in retention in care at 12 months in the intervention group compared to the controls. The use of a combination of post-diagnosis linkage strategies, therefore, has the potential of improving linkage to care. 


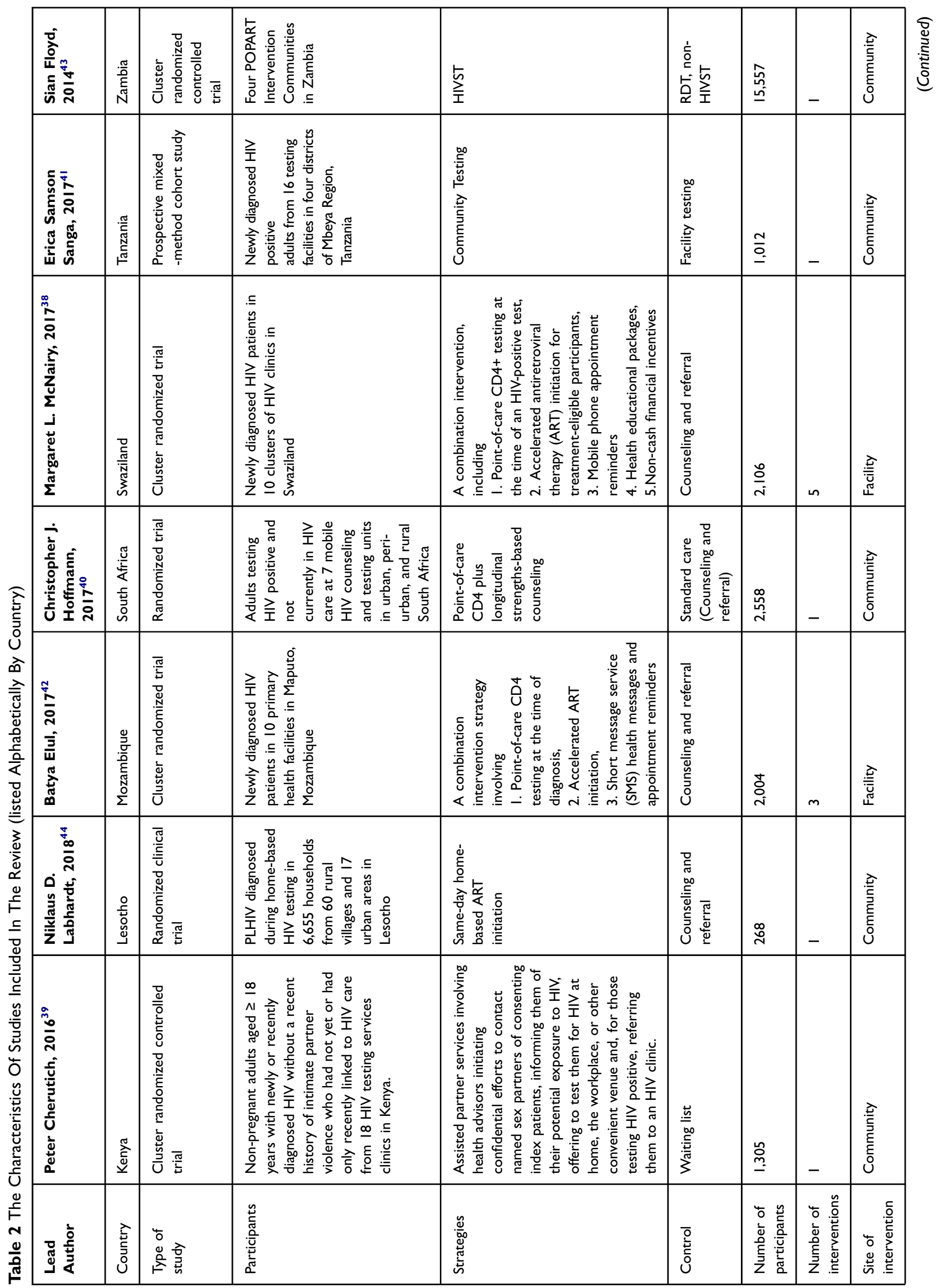




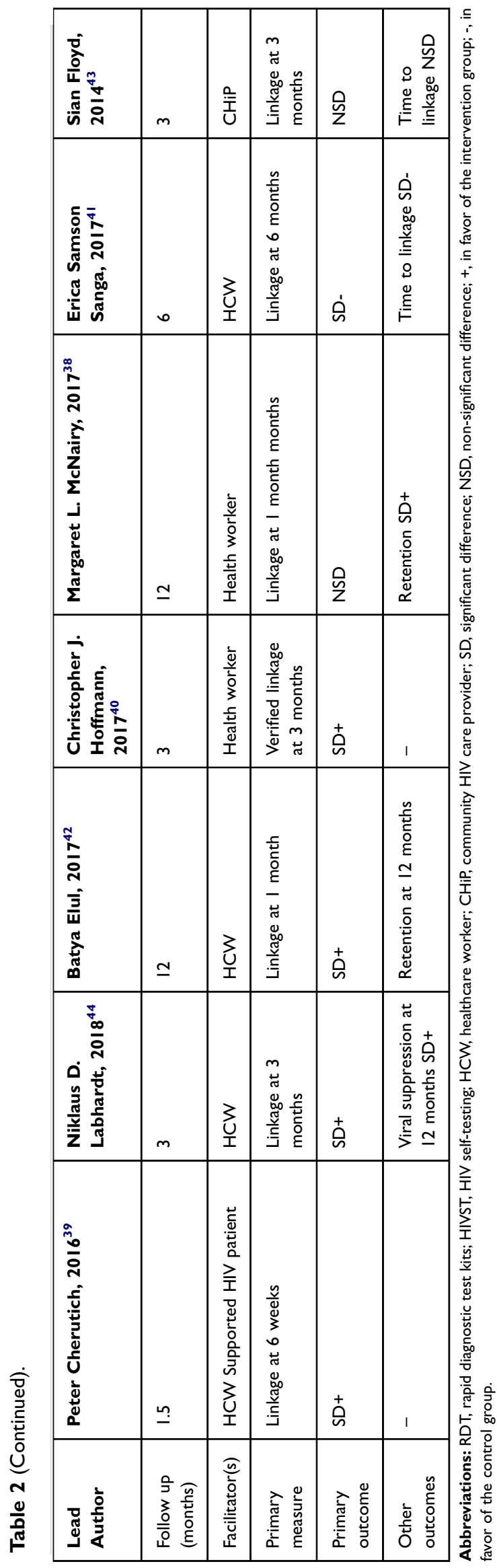

\section{- Task shifting}

All but one of the strategies in the included studies were delivered by Health care workers. Floyd et al assessed the impact of HIVST on linkage to care, in a cluster randomized intervention delivered by Community HIV Care Providers (CHiPs) in Zambia. ${ }^{43}$ Three months after diagnosis, there was no significant difference in the linkage rates between the intervention and non-intervention settings $(\mathrm{HR}=1.12$, 95\% CI $=0.8-1.6$ ) whether HIVST was supervised or not. Even though the CHiPs have reportedly supported linkage in a number of studies, their failure to make an impact in this intervention might be due to the testing method used.

\section{Patient Convenience And Accessibility \\ - Immediate CD4 count testing}

The most popular of these is the POC CD4 testing, which was used as part of a combination of strategies conducted in Mozambique, South Africa, and Swaziland ${ }^{38,40,42}$ and as a strategy on its own. ${ }^{40}$ This strategy failed to demonstrate a significant impact on linkage in the combination intervention by McNairy et $\mathrm{al}^{38}(\mathrm{RR}=1.48, \mathrm{CI}: 1.18 \pm 1.86$, $p=0.002)$, and in combination with care facilitation as assessed by Hoffman et al. ${ }^{40}$ (HR=1.4, CI:1.1 to 1.7, $p=0.001$ ). However, it significantly improved linkage as part of other strategies in the study by Elul et $\mathrm{al}^{42}$ $(\mathrm{RR}=1.48, \mathrm{CI}: 0.93 \pm 2.35, \quad p=0.09)$ and on its own $(\mathrm{HR}=1.0,95 \% \mathrm{CI}=0.86$ to 1.3$)$. Therefore, POC CD4 testing has the potential to improve linkage to care.

- Immediate ART initiation

The impact of accelerated ART initiation on linkage was also assessed as part of two combination strategies in Swaziland and Mozambique ${ }^{38,42}$ and as an intervention on its own in Lesotho. ${ }^{44}$ On its own, it significantly improved linkage to care (absolute difference, $25.6 \%$; $95 \%$ CI, $13.8 \%$ to $36.3 \%$; $p<0.001)$ at 3 months post-diagnosis and also improved linkage significantly one month after diagnosis in one of the multiple strategy or combination interventions. ${ }^{42}$ Therefore, it has the potential to be used as a strategy to improve linkage to care.

\section{- Community HIV testing}

The impact of Community HIVST and mobile HIV testing on linkage to care was assessed by Floyd et al in Zambia ${ }^{43}$ 
and Sanga et al in Tanzania. ${ }^{41}$ Both strategies, however, failed to significantly improve linkage to care. In Tanzania, $84 \%$ (CI $81 \%$ to $87 \%, n=512$ ) of individuals tested at the facility-based site were linked to care compared to $69 \%$ (CI $65 \%$ to $74 \%, n=281$ ) of individuals tested at mobile site 6 months after diagnosis. ${ }^{41}$ Likewise, in Zambia, 65\% of individuals tested at HIVST zones compared to $64 \%$ in non-HIVST Zones were linked to care 3 months after diagnosis $(\mathrm{HR}=1.12,95 \% \mathrm{CI}=0.8-1.6) .{ }^{43}$ Community HIV testing strategies as stand-alone interventions may therefore not be recommended as linkage improving modalities.

\section{Behavior Interventions And Patient Support \\ - Assisted partner services}

The impact of assisted partner services, as described in the introduction, was assessed by Cherutich et al in Kenya. ${ }^{39}$ Compared to the waiting list controls, a significant proportion, $15 \%$ of the intervention group versus $3 \%$ of the controls were newly enrolled in care $(\mathrm{IRR}=4 \cdot 4, \mathrm{CI}=2 \cdot 6-7 \cdot 4)$. This could, therefore, be explored as a

\section{- Care facilitation}

This was a five-session counseling strategy delivered by Hoffman et $\mathrm{al}^{40}$ as part of a multiple strategy or combination intervention including POC CD4 testing in South Africa. This strategy, however, failed to demonstrate a significant improvement in the verified linkage, compared to the standard care controls 3 months after diagnosis ( $\mathrm{HR}=1.4, \mathrm{CI}=1.1$ to $1.7, p=0.001)$. Multiple counseling sessions after diagnosis may therefore not be needed to improve linkage to care.

- Mobile phone appointment reminders and health education,

Elul et $\mathrm{al}^{42}$ and McNairy et al ${ }^{38}$ employed mobile phone appointment reminders and health education as part of a combination of strategies to improve linkage. ${ }^{38,42}$ Whilst the strategies delivered in Mozambique demonstrated a significant improvement in linkage one month after diagnosis $(\mathrm{RR}=1.48, \mathrm{CI}: 0.93 \pm 2.35, p=0.09),{ }^{42}$ the one in Swaziland failed to do so after the same period of observation $(\mathrm{RR}=1.48, \mathrm{CI}: 1.18 \pm 1.86, p=0.002) .{ }^{38}$ There may be the need to explore the use of mobile phone appointment reminders and health education as stand-alone interventions in this setting to assess its impact on the linkage.

\section{Use Of Incentives}

McNairy et al used non-cash financial incentives (mobile airtime) as part of a multiple strategy intervention in Swaziland. One month after diagnosis, there was no significant difference in the linkage between comparison arms $(\mathrm{RR}=1.48, \mathrm{CI}: 1.18$ $\pm 1.86, p=0.002) .{ }^{38}$ Hoffman et al also observed that the addition of transport reimbursement to POC CD4 did not make any significant improvement in linkage as both the POC alone and POC in addition to the transport reimbursement arm both had $31 \%$ verified linkage 3 months after diagnosis. ${ }^{40}$ The use of cash and non-cash incentives as linkage strategies may therefore not be recommended.

\section{Impact Of The Intervention Site On The Linkage}

From Table 2 (Details of included studies), one of the two interventions delivered in health facilities and three out of the five interventions delivered in the community setting were associated with a significant improvement in the linkage to care.

\section{Impact Of The Intervention Facilitator On Linkage}

Four out of the six interventions with strategies delivered by health workers were associated with a significant improvement in linkage but the only intervention strategy delivered by lay providers (CHiPs) did not make a significant difference. Therefore, there is a need to use health workers to deliver linkage strategies in the interventions.

\section{Impact On Other Outcomes Retention At 12 Months}

The two included studies that used a combination of linkage strategies, ${ }^{38,42}$ led to significant retention at 12 months in the intervention arm as compared to the controls. Only one, however, reported a significant linkage rate. The use of multiple linkage strategies in an intervention, therefore, has the potential to improve both linkage and retention in care

\section{Time To Linkage}

Two studies assessed time to linkage. Whilst Floyd et al ${ }^{43}$ recorded no significant difference in time to linkage between the comparison arms after the use of HIVST, Sanga et $\mathrm{al}^{41}$ noticed a worse time to linkage for their community testing intervention arm as compared to the 
facility testing controls. The strategies in the intervention, therefore, failed to significantly shorten the time to linkage.

\section{Viral Suppression}

Labhardt et $\mathrm{al}^{44}$ reported a significant improvement in viral suppression at 12 months following same-day home-based ART delivery to newly diagnosed individuals.

\section{Quality Of Included Studies}

This was assessed using the Cochrane collaborations tool for assessing the risk of bias $^{46}$ and the Newcastle-Ottawa scale for observational studies. The results are summarized below:

\section{Sequence Generation}

Six of the included studies ${ }^{38-42,44}$ described their methods of sequence generation but that of Floyd et $\mathrm{al}^{43}$ was unclear. Apart from Elul et al. ${ }^{42}$ all the other studies had a low risk of bias in sequence generation and used either random number tables or a computer-generated sequence.

\section{Allocation Concealment}

Whilst two of the included studies had an unclear risk of bias in allocation concealment, only two of the five remaining studies also had a low risk of bias due to the use of sealed opaque envelopes and central allocation.

\section{Blinding}

Apart from Hoffman et $\mathrm{al}^{40}$ that had a low risk of bias in blinding, the rest of the included studies had an unclear risk of bias.

\section{Incomplete Outcome Data}

A total of 5 out of the 7 included studies had a low risk of bias by the use of imputation techniques such as the last observation carried forward, multiple imputation analysis, and mixed model analysis. However, Sanga et $\mathrm{al}^{41}$ and Floyd et $\mathrm{al}^{43}$ had an unclear risk of bias in incomplete outcome data.

\section{Selective Outcome Reporting}

Apart from Hoffman et $\mathrm{al}^{40}$ that published their study protocol, and had a low risk of bias from selective outcome reporting, the assessment of this bias from the other studies was deemed unclear.

\section{Other Sources Of Bias}

There were other sources of bias in all the included studies that might have influenced the results. These included lack of power due to small sample sizes and significant differences in baseline characteristics in the comparison arms. A summary of the risk of bias assessment can be found in Appendix C.

\section{Discussion}

This review sought to synthesize the evidence on strategies implemented to bridge the barriers in linking newly diagnosed HIV patients to care in urban areas of sub-Saharan Africa. The strategies in the 7 identified studies could be categorized under health system interventions, patient convenience and accessibility, behavior interventions and patient support, and incentives as identified by Govindasamy et $\mathrm{al}^{25}$ Even though the use of various incentives failed to improve linkage in the included studies, some strategies under the other domains have the potential to improve linkage to care.

Similarly, Floyd et al found home-based HIV self-testing (HIVST) not to be an effective linkage strategy, however, a systematic review by Mavegam et $\mathrm{al}^{47}$ found it effective in improving linkage to care. Monisha et $\mathrm{al}^{48}$ in their review, also found home-based HIVST to be effective in reaching and linking a high proportion of young adults. This difference in results might be due to a difference in the target age groups with the two successful strategies focusing on those aged 15-24 years, a subset of patients that Floyd et al assessed.

Moreover, the use of lay workers to deliver HIVST may also have accounted for the finding by Floyd et $\mathrm{al}^{43}$ even though they were instrumental in the success of the Bukoba Combination Prevention, intervention (BCPI) in Tanzania, that recorded a $90 \%$ linkage rate, 3 months after diagnosis. $^{49}$ In the BCPI, the HIV positive patients in the intervention arm received a package of lay worker delivered linkage services recommended by WHO, the International Association of Providers of AIDS Care (IAPAC) and the U.S. Centers for Disease Control and Prevention (CDC). Lay workers may, therefore, be potentially useful in the delivery of other linkage strategies apart from HIVST. However, there will be the need to develop a structured plan to guide their orientation and community intervention delivery as was done in the BCPI. McNairy et al also implemented a combination approach using multiple linkage strategies which did not significantly improve linkage. The use of evidence-based recommendations as done in the BCPI could have improved their outcomes.

A systematic review and a meta-analysis by Croxford et al on linkage to HIV care following diagnosis in the WHO European region showed that the use of SMS as a standalone strategy significantly reduced the time to linkage and 
improved linkage rates. ${ }^{7}$ This confirms the potential benefits of the use of this cheap technology as a linkage strategy. However, there may the need for further trials in the urban sub-Saharan Africa region to assess its impact as a stand-alone strategy.

Mavegam et al found community or mobile HIV counseling and testing to be an effective linkage strategy as opposed to the finding by Sanga et al in this review. Their success might be due to the active referral of their newly diagnosed patients during community testing to health facilities by the one who performed the test. Of all the linkage approaches examined by Monisha et al, they found mobile testing to have reached and linked the highest proportion of men. Community testing may, therefore, be potentially useful, especially for men if resources are made available to support active referrals after diagnosis.

Though a randomized controlled trial by El Sadr et $\mathrm{al}^{50}$ in the United States agreed with two of the studies included in this review by McNair et al and Hoffman et al that incentives do not make a significant impact on improving linkage rates, a systematic review by Basset et $a^{51}$ concluded that financial incentives show promise for improving engagement across the cascade of HIV care. Their review, however, had significant limitations in the use of mostly under-powered studies, which were conducted in different contexts. A systematic review of the effectiveness of financial incentives on linkage in urban Sub-Saharan Africa needs to be conducted. This will help test concepts from Behavioral Economics which as postulated, "may help improve engagement in HIV care by addressing upstream structural risk factors for HIV, such as poverty, or by providing conditional rewards for immediate, measurable outcomes related to HIV care". 51 Until then, the use of financial incentives as a linkage strategy in urban settings in the sub-region may not be advisable.

Of the three behavior and patient support intervention strategies reported in this review, the only one that significantly improved linkage was implemented as a stand-alone intervention. This is supported by the review by Anderson et $\mathrm{al}^{52}$ that behavioral intervention strategies at the community level are better delivered as stand-alone strategies rather than as part of other strategies. It might, however, be important to conduct systematic reviews and trials in the sub-region to compare the impact of stand-alone and combination behavioral strategies on linkage to care in order to make a more robust recommendation on the best approach to use.

Though the two studies reporting time to linkage could not demonstrate significant improvement, Mavegam et al found the use of referral forms, provision of transportation allowance, home initiation of HIV care, and volunteer escort to the HIV care units to be effective in reducing time to linkage to care. Home initiation of HIV care has already been reported in this review to be a good linkage strategy. The effect of this strategy to reduce time to linkage and improve linkage rates makes it the strategy of choice in the urban settings of sub-Saharan Africa.

The review has a number of limitations including the constraints of time and use of a data extraction form that was not piloted which could lead to selection and information bias. Though a comprehensive search was done, including both published and grey literature, limited access to critical databases as well as lack of the use of truncation, wildcard and proximity measures might have limited the number of quality studies retrieved. The probability of publication bias, ${ }^{53}$ is likely in the included studies because most of them reported favorable outcomes. The definitions of outcomes were heterogeneous across included studies and therefore, effects of similar or different strategies were difficult to compare.

\section{Conclusions}

Assisted partner services, same day home-based ART initiation, combination intervention strategies and POC CD4 testing significantly improved linkage to care in urban settings of the sub-Sahara African region. They can be delivered either in a health facility or in the community but should be facilitated by health workers. There is, however, the need to conduct more linkage specific studies in the sub-region to assess the use of financial incentives and stand-alone versus combination intervention strategies in improving linkage.

\section{Disclosure}

The authors reports no conflicts of interest in this work.

\section{References}

1. WHO. WHO | HIV/AIDS. WHO. 2018. Available from: https://www. who.int/gho/hiv/en/. Accessed July 23, 2019.

2. UNAIDS. UNAIDS gap report; 2014. ISBN 978-92-9253-062-4.

3. World Health Organisation. Who | HIV/AIDS. WHO; 2014.

4. UNAIDS. Global HIV statistics 2017. Jt United Nations Program HIV/AIDS. 2018;3:5. Available from: http://www.unaids.org/sites/ default/files/media_asset/UNAIDS_FactSheet_en.pdf.

5. WHO. WHO | HIV/AIDS Prevalence in Sub-Saharan Africa. WHO; 2016.

6. UNAIDS. 90-90-90 An ambitious treatment target to help end the AIDS epidemic. http://WwwUnaidsOrg/Sites/Default/Files/Media Asset/90-90-90_En_0Pdf.2014:40. doi:10.7448/IAS.16.4.18751.

7. Croxford S, Yin Z, Burns F, et al. Linkage to HIV care following diagnosis in the WHO European Region: a systematic review and metaanalysis, 2006-2017. PLoS One. 2018;13(2):e0192403. doi:10.1371/ journal.pone. 0192403 
8. UNAIDS/WHO. UNAIDS Report on the Global AIDS Epidemic; 2012. Available from: https://www.unaids.org/sites/default/files/ media_asset/20121120_UNAIDS_Global_Report_2012_with annexes_en_1.pdf. Accessed July 23, 2019.

9. Johnson AS, Hess K, Hu S, et al. Monitoring Selected National HIV Prevention and Care Objectives by Using HIV Surveillance DataUnited States and 6 Dependent Areas, 2015. Vol. 22; 2017. Available from: http://www.cdc.gov/hiv/library/reports/hiv-surveillance.html. http://www.cdc.gov/hiv/library/reports/hiv-surveillance.htmlhttp:// www.cdc.gov/cdc-info/requestform.html. Accessed December 2, 2018.

10. Hall HI, Halverson J, Wilson DP, et al. Late diagnosis and entry to care after diagnosis of human immunodeficiency virus infection: a country comparison. Palaniyar N, ed. PLoS One. 2013;8(11):e77763. doi:10.1371/journal.pone.0077763

11. Spach DH, Kinney RG. Core concepts - HIV diagnostic testing Screening and diagnosis - National HIV curriculum. Available from: https://www.hiv.uw.edu/go/screening-diagnosis/linkage-care/core-con cept/all. Accessed December 2, 2018.

12. Cohen MS, Chen YQ, McCauley M, et al. Prevention of HIV-1 infection with early antiretroviral therapy. $N$ Engl J Med. 2011;365 (6):493-505. doi:10.1056/NEJMoa1105243

13. McNairy ML, El-Sadr WM. Antiretroviral Therapy For The Prevention of HIV Transmission: what will it take? Clin Infect Dis. 2014;58(7):1003-1011. doi:10.1093/cid/ciu018

14. Fox MP, Shearer K, Maskew M, Meyer-Rath G, Clouse K, Sanne I. Attrition through multiple stages of pre-treatment and ART HIV care in South Africa. PLoS One. 2014;9(10):e110252. doi:10.1371/journal.pone. 0110252

15. Fox MP, Rosen S. Retention of adult patients on antiretroviral therapy in low- and middle-income countries. J Acquir Immune Defic Syndr. 2015;69(1):98-108. doi:10.1097/QAI.0000000000000553

16. Iwuji CC, Orne-Gliemann J, Larmarange J, et al. Uptake of homebased HIV testing, linkage to care, and community attitudes about ART in rural KwaZulu-Natal, South Africa: descriptive results from the first Phase of the ANRS 12249 TasP cluster-randomised trial Low N, ed. PLoS Med. 2016;13(8):e1002107. doi:10.1371/journal. pmed.1002107

17. McNairy ML, Lamb MR, Abrams EJ, et al. Use of a comprehensive HIV care cascade for evaluating HIV program performance. J Acquir Immune Defic Syndr. 2015;70(2):e44-e51. doi:10.1097/QAI.0000000000000745

18. Mugglin C, Estill J, Wandeler G, et al. Loss to programme between HIV diagnosis and initiation of antiretroviral therapy in sub-Saharan Africa: systematic review and meta-analysis. Trop Med Int Heal. 2012;17(12):1509-1520. doi:10.1111/j.1365-3156.2012.03089.x

19. UNAIDS. UNAIDS Report on the Global AIDS Epidemic 2013 Global Report; 2013. Available from: https://www.unaids.org/sites/ default/files/media_asset/UNAIDS_Global_Report_2013_en_1.pdf. Accessed July 23, 2019.

20. Fairall LR, Bachmann MO, Louwagie GMC, et al. Effectiveness of antiretroviral treatment in a South African program: a cohort study. Arch Intern Med. 2008;168(1):86. doi:10.1001/archinternmed.2007.10

21. WHO 9.3 Retention across the continuum of care. WHO; 2013. Available from: http://www.who.int/hiv/pub/guidelines/arv2013/ operational/retention/en/. Accessed December 2, 2018.

22. Fox MP, Rosen S. Patient retention in antiretroviral therapy programs up to three years on treatment in sub-Saharan Africa, 2007-2009: systematic review. Trop Med Int Heal. 2010;15:1-15. doi:10.1111/ j.1365-3156.2010.02508.x

23. Rosen S, Fox MP, Bartlett J. Retention in HIV care between testing and treatment in sub-Saharan Africa: a systematic review. Bartlett J, ed. PLoS Med. 2011;8(7):e1001056. doi:10.1371/journal.pmed.1001056

24. Braitstein P, Brinkhof MWG, Dabis F, et al. Mortality of HIV-1infected patients in the first year of antiretroviral therapy: comparison between low-income and high-income countries. Lancet. 2006;367 (9513):817-824. doi:10.1016/S0140-6736(06)68337-2
25. Govindasamy D, Meghij J, Negussi EK, Baggaley RC, Ford N, Kranzer K. Interventions to improve or facilitate linkage to or retention in pre-ART (HIV) care and initiation of ART in low- and middleincome settings - A systematic review. J Int AIDS Soc. 2014;17:19032. doi:10.7448/IAS.17.1.19032

26. Bekker LG, Montaner J, Ramos C, et al. IAPAC guidelines for optimizing the HIV care continuum for adults and adolescents. $J$ Int Assoc Provid AIDS Care. 2015;14:S3-S34. doi:10.1177/2325 957415613442

27. Christopoulos KA, Kaplan B, Dowdy D, et al. Testing and linkage to care outcomes for a clinician-initiated rapid HIV testing program in an urban emergency department. AIDS Patient Care STDS. 2011;25 (7):439-444. doi:10.1089/apc.2011.0041

28. Wanyenze RK, Hahn JA, Liechty CA, et al. Linkage to HIV care and survival following inpatient HIV counseling and testing. AIDS Behav. 2011;15(4):751-760. doi:10.1007/s10461-010-9704-1

29. Kitahata MM, Gange SJ, Abraham AG, et al. Effect of early versus deferred antiretroviral therapy for HIV on survival. $N$ Engl $J$ Med. 2009;360(18):1815-1826. doi:10.1056/NEJMoa0807252

30. Bocour A, Renaud TC, Udeagu C-CN, Shepard CW. HIV partner services are associated with timely linkage to HIV medical care. AIDS. 2013;27(18):2961-2963. doi:10.1097/QAD.0000000000000031

31. Centers for Disease Control. Recommendations for partner services programs for HIV infection, syphilis, gonorrhea, and chlamydial infection. MMWR Recomm Rep Morb Mortal Wkly Rep. 2008;57 (RR-9):1-83; quiz CE1-4. doi:10.2307/42001003

32. Greene E, Pack A, Stanton J, et al. "It makes you feel like someone cares" acceptability of a financial incentive intervention for HIV viral suppression in the HPTN 065 (TLC-Plus) study. Ferrand RA, ed. PLoS One. 2017;12(2):e0170686. doi:10.1371/ journal.pone. 0170686

33. UNAIDS. Turning point for Africa - An historic opportunity to end AIDS as a public health threat by 2030 and launch a new era of sustainability. UNAIDS.

34. The World Bank. Sub-Saharan Africa Data. doi:10.1016/j.gheart. 2018.09.514

35. World Health Organization. Patient Monitoring Guidelines for HIV Care and Antiretroviral Therapy (ART). World Health Organization; 2006.

36. Wells GA, Shea B, O'Connell D, Peterson J, Welch VM, Losos PT. Ottawa Hospital Research Institute. Available from: http://www.ohri.ca/programs/ clinical_epidemiology/oxford.asp. Accessed December 2, 2018.

37. Borek AJ, Abraham C, Smith JR, Greaves CJ, Tarrant M. A checklist to improve reporting of group-based behaviour-change interventions. BMC Public Health. 2015;15(1):963. doi:10.1186/ s12889-015-2300-6

38. McNairy ML, Lamb MR, Gachuhi AB, et al. Effectiveness of a combination strategy for linkage and retention in adult HIV care in Swaziland: the Link4Health cluster randomized trial. Deeks SG, ed. PLoS Med. 2017;14(11):e1002420. doi:10.1371/journal.pmed. 1002420

39. Cherutich P, Golden MR, Wamuti B, et al. Assisted partner services for HIV in Kenya: a cluster randomised controlled trial. Lancet HIV. 2017;4(2):e74-e82. doi:10.1016/S2352-3018(16)30214-4

40. Hoffmann CJ, Mabuto T, Ginindza S, et al. Strategies to accelerate HIV care and antiretroviral therapy initiation after HIV diagnosis. $J$ Acquir Immune Defic Syndr. 2017;75(5):540-547. doi:10.1097/QAI. 0000000000001428

41. Sanga ES, Lerebo W, Mushi AK, et al. Linkage into care among newly diagnosed HIV-positive individuals tested through outreach and facility-based HIV testing models in Mbeya, Tanzania: a prospective mixed-method cohort study. BMJ Open. 2017;7(4):e013733. doi:10.1136/bmjopen-2016-013733

42. Elul B, Lamb MR, Lahuerta M, et al. A combination intervention strategy to improve linkage to and retention in HIV care following diagnosis in Mozambique: a cluster-randomized study. Lewin SR, ed. PLoS Med. 2017;14(11):e1002433. doi:10.1371/journal. pmed.1002433 
43. Floyd S, Shanaube K, Schaap A, et al. Linkage to HIV care following HIV self-testing: results from a cluster-randomised trial of community-based distribution of oral HIV self-test kits nested in four HPTN 071 (PopART) communities in Zambia. https://programme.aids2018. org//PAGMaterial/eposters/7432.pdf. Accessed December 8, 2018.

44. Labhardt ND, Ringera I, Lejone TI, et al. Effect of offering same-day ART vs usual health facility referral during home-based HIV testing on linkage to care and viral suppression among adults with HIV in Lesotho: the CASCADE randomized clinical trial. JAMA. 2018;319 (11):1103-1112. doi:10.1001/jama.2018.1818

45. Ayles H, Floyd S, Mulubwa C, et al. Increasing knowledge of HIV status among Men: a cluster-randomised trial of community-based distribution of oral HIV self-test kits nested in four HPTN 071 communities in Zambia. IAS; 2017.

46. Cochrane Bias Methods Group. Assessing Risk of Bias in Included Studies. Cochrane Bias; 2016.

47. Mavegam BO, Pharr JR, Cruz P, Ezeanolue EE. Effective interventions to improve young adults' linkage to HIV care in sub-Saharan Africa: a systematic review. AIDS Care. 2017;29(10):1198-1204. doi:10.1080/09540121.2017.1306637
48. Sharma M, Ying R, Tarr G, Barnabas R. Systematic review and metaanalysis of community and facility-based HIV testing to address linkage to care gaps in sub-Saharan Africa. Nature. 2015;528 (7580):S77. doi:10.1038/nature16044

49. Pepfar. Bukoba Combination Prevention Evaluation: Effective Approaches to Linking People Living with HIV to Care and Treatment Services in Tanzania - PEPFAR Solutions Platform (BETA).

50. El-Sadr WM, Donnell D, Beauchamp G, et al. Financial incentives for linkage to care and viral suppression among HIV-positive patients. JAMA Intern Med. 2017;177(8):1083. doi:10.1001/ jamainternmed.2017.2158

51. Bassett IV, Wilson D, Taaffe J, Freedberg KA. Financial incentives to improve progression through the HIV treatment cascade. Curr Opin HIV AIDS. 2015;10(6):451-463. doi:10.1097/COH.0000000000000196

52. Anderson NB, Bulatao RA, Cohen B. National Research Council (US) Panel on Race $\mathrm{E}$ and $\mathrm{H}$ in LL. Behavioral Health Interventions: What Works and Why? 2004.

53. Lecture 5B. Selection Bias - Johns Hopkins University. Coursera.

\section{Publish your work in this journal}

HIV/AIDS - Research and Palliative Care is an international, peerreviewed open-access journal focusing on advances in research in HIV, its clinical progression and management options including antiviral treatment, palliative care and public healthcare policies to control viral spread. The manuscript management system is completely online and includes a very quick and fair peer-review system, which is all easy to use. Visit http://www.dovepress.com/testimonials.php to read real quotes from published authors. 